\title{
Stabilization of a Bose-Einstein droplet by hyperfine Rabi oscillations
}

\author{
Hiroki Saito, ${ }^{1}$ Randall G. Hulet, ${ }^{2}$ and Masahito Ueda ${ }^{3,4}$ \\ ${ }^{1}$ Department of Applied Physics and Chemistry, The University of Electro-Communications, Tokyo 182-8585, Japan \\ ${ }^{2}$ Department of Physics and Astronomy and Rice Quantum Institute, Rice University, Houston, Texas 77251, USA \\ ${ }^{3}$ Department of Physics, Tokyo Institute of Technology, Tokyo 152-8551, Japan \\ ${ }^{4}$ ERATO Macroscopic Quantum Control Project, JST, Tokyo 113-8656, Japan
}

(Received 30 July 2007; published 21 November 2007)

\begin{abstract}
A self-trapped Bose-Einstein condensate is shown to be stabilized in two-dimensional free space by Rabi oscillations between two hyperfine states which make an effective interatomic interaction oscillate in time. The stabilization mechanism is elucidated by using a two-component Gross-Pitaevskii equation combined with a variational analysis. The parameter regime of stability is investigated.
\end{abstract}

DOI: 10.1103/PhysRevA.76.053619

PACS number(s): 03.75.Lm, 03.75.Kk, 03.75.Mn, 05.45.Yv

\section{INTRODUCTION}

A droplet of water is a self-trapped object, in which attractive and repulsive interactions between the water molecules are balanced. Such a self-trapped system is hard to achieve in the usual gaseous phase, since thermal expansion of the gas must be prevented by a strong attractive interaction between particles, which, however, would lead to collapse of the gas into a denser phase. A gaseous Bose-Einstein condensate (BEC) is the most promising candidate for creating a self-trapped gas, or a "BEC droplet," because of unprecedented controllability of the parameters of the system.

In one dimension (1D), a BEC droplet, or a matter-wave bright soliton, is stable with an attractive interaction between atoms counterbalancing zero-point quantum pressure. This novel state of a gas has been experimentally achieved by the ENS group [1] and the Rice group [2] by using a ${ }^{7} \mathrm{Li}$ condensate in quasi-1D traps. In $2 \mathrm{D}$ and higher dimensions, however, there is neither a stable nor metastable state of a BEC droplet if the interaction is short range and constant in time [3].

Dynamic stabilization is a possible way to obtain a BEC droplet in 2D and 3D. It has been shown that if the interaction is made to oscillate in time, a BEC droplet can be dynamically stabilized in $2 \mathrm{D}[4,5]$ by a mechanism similar to that for the stabilization of an inverted pendulum [6]. This stabilization mechanism for a BEC droplet has been studied by several authors [7-12]. We have shown that the same mechanism can also stabilize a BEC droplet in 3D in the presence of dissipation [13]. Another dynamic scheme to stabilize a BEC droplet in 3D is to use feedback control of the interaction [14].

With respect to the stabilization method proposed in Refs. $[4,5]$, we must oscillate the interaction between repulsive and attractive at a frequency much higher than the characteristic frequencies of the system. If we use the magnetic Feshbach resonance [15] to oscillate the interaction, we should oscillate the strength of the applied magnetic field at such a high frequency. We propose in the present study a method to achieve this dynamic stabilization without using any timedependent Feshbach control of the interaction. Our idea is to use two hyperfine states with different scattering lengths. We will show that the Rabi oscillation between these hyperfine states causes an effective oscillation of the scattering length, thereby stabilizing a BEC droplet through the mechanism proposed in Refs. [4,5]. This method simply irradiates the system with a constant electromagnetic wave.

This paper is organized as follows. Section II is devoted to describing a system of a two-component BEC driven by an external field. Section III performs numerical integration of the two-component Gross-Pitaevskii equation to show that the BEC droplet is indeed dynamically stabilized. The stability diagram for the scattering lengths is obtained. Section IV derives an effective single-component equation and combines it with a variational method to elucidate the stabilization mechanism for a BEC droplet by hyperfine Rabi oscillations. Section V provides conclusions.

\section{FORMULATION OF THE PROBLEM}

We consider a situation in which bosonic atoms with hyperfine degrees of freedom are irradiated by a uniform electromagnetic wave that is resonant with the energy difference between two hyperfine states of the atoms. The atoms are assumed to be tightly confined in the $z$ direction by harmonic potential $m \omega_{z} z^{2} / 2$ that is independent of the hyperfine state. If $\hbar \omega_{z}$ is much larger than the other characteristic energy scales, the wave function in the $z$ direction is frozen in the ground state of the harmonic potential, and then the system can be reduced to $2 \mathrm{D}$. We will consider the $2 \mathrm{D}$ system in the subsequent analysis.

The Hamiltonian for the system consists of three parts:

$$
\hat{H}=\hat{H}_{0}+\hat{H}_{\mathrm{f}}+\hat{H}_{\text {int }} \text {. }
$$

The noninteracting part of the Hamiltonian $\hat{H}_{0}$ is given by

$$
\hat{H}_{0}=\int d \boldsymbol{r} \sum_{i=1}^{2} \hat{\psi}_{i}^{\dagger}(\boldsymbol{r})\left[-\frac{\hbar^{2}}{2 m} \nabla^{2}+V_{i}(\boldsymbol{r})\right] \hat{\psi}_{i}(\boldsymbol{r}),
$$

where $\hat{\psi}_{i}(\mathbf{r})$ annihilates an atom in hyperfine state $i, m$ is the atomic mass, and $V_{i}(\mathbf{r})$ is an external trapping potential for hyperfine state $i$. The effect of the resonant field on atomic state is described by 


$$
\hat{H}_{\mathrm{f}}=\hbar \int d \boldsymbol{r}\left[\Omega \hat{\psi}_{1}^{\dagger}(\boldsymbol{r}) \hat{\psi}_{2}(\boldsymbol{r})+\Omega^{*} \hat{\psi}_{2}^{\dagger}(\boldsymbol{r}) \hat{\psi}_{1}(\boldsymbol{r})\right]
$$

where $|\Omega|$ is the Rabi frequency and $\arg (\Omega)$ is the phase of the field. The interaction between ultracold atoms is short range and described by the contact Hamiltonian

$$
\hat{H}_{\mathrm{int}}=\frac{2 \pi \hbar^{2}}{m} \sqrt{\frac{m \omega_{z}}{2 \pi \hbar}} \int d \boldsymbol{r} \sum_{i, j=1}^{2} a_{i j} \hat{\psi}_{i}^{\dagger}(\boldsymbol{r}) \hat{\psi}_{j}^{\dagger}(\boldsymbol{r}) \hat{\psi}_{j}(\boldsymbol{r}) \hat{\psi}_{i}(\boldsymbol{r}),
$$

where $a_{i j}$ is the $s$-wave scattering length between atoms in hyperfine states $i$ and $j$. The factor $\left[m \omega_{z} /(2 \pi \hbar)\right]^{1 / 2}$ in Eq. (4) comes from the integration with respect to $z$.

In the mean-field approximation, the system at zero temperature is described by the 2D Gross-Pitaevskii (GP) equations

$$
\begin{aligned}
i \hbar \frac{\partial \psi_{1}}{\partial t}= & \left(-\frac{\hbar^{2}}{2 m} \nabla^{2}+V_{1}\right) \psi_{1}+\hbar \Omega \psi_{2}+g_{11}\left|\psi_{1}\right|^{2} \psi_{1} \\
& +g_{12}\left|\psi_{2}\right|^{2} \psi_{1}, \\
i \hbar \frac{\partial \psi_{2}}{\partial t}= & \left(-\frac{\hbar^{2}}{2 m} \nabla^{2}+V_{2}\right) \psi_{2}+\hbar \Omega^{*} \psi_{1}+g_{22}\left|\psi_{2}\right|^{2} \psi_{2} \\
& +g_{12}\left|\psi_{1}\right|^{2} \psi_{2},
\end{aligned}
$$

where $\psi_{i}(\mathbf{r}, t)$ is the macroscopic wave function satisfying the normalization condition

$$
\int d \boldsymbol{r} \sum_{i=1}^{2}\left|\psi_{i}(\boldsymbol{r}, t)\right|^{2}=N
$$

with $N$ being the number of atoms, and

$$
g_{i j}=\frac{4 \pi \hbar^{2} a_{i j}}{m} \sqrt{\frac{m \omega_{z}}{2 \pi \hbar}}
$$

representing the interaction coefficients.

\section{STABILIZATION OF A TWO-COMPONENT BEC DROPLET}

The interaction parameter of the system oscillates between $g_{11}$ and $g_{22}$ by the Rabi oscillation between two hyperfine states. If one of them is positive and the other is negative, the stabilization mechanism in Refs. [4,5] is expected to be applicable to the present system. This is the main idea of the present paper.

We assume that all the atoms are initially in hyperfine state 1 and confined in radial trapping potential $V_{1}$ $=m \omega_{\perp}^{2} r^{2} / 2$. The initial wave function $\psi_{1}$ is then the ground state of GP equation (5a) with $\psi_{2}=0$ and $\Omega=0$. At $t=0$, the radial trapping potential is switched off and the radiation field is switched on-i.e., $V_{1}=V_{2}=0$ and $\Omega \neq 0$ for $t>0$.

We first investigate the dynamics of the system for specific values of the scattering lengths: $a_{11}=0.75 \mathrm{~nm}, a_{22}=$ $-1.5 \mathrm{~nm}$, and $a_{12}=0$. The radial trapping frequency for preparing the initial state is $\omega_{\perp}=2 \pi \times 25 \mathrm{~Hz}$, and the trapping (a)

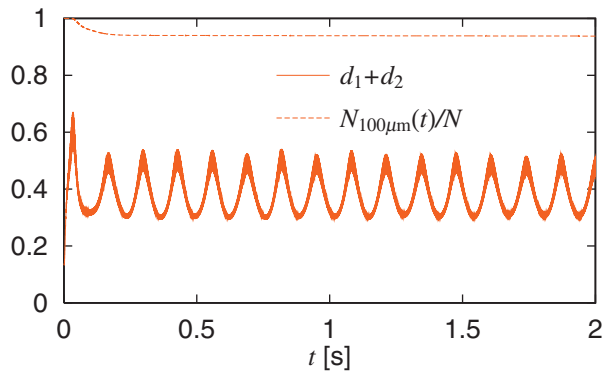

(b)

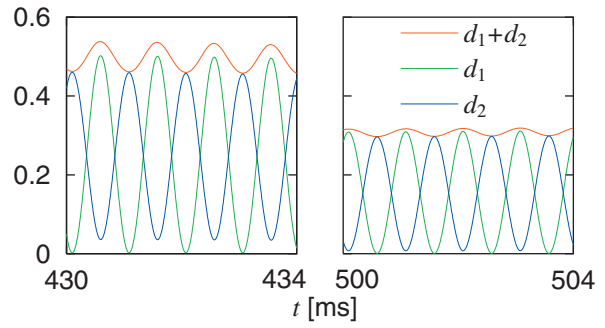

(c)
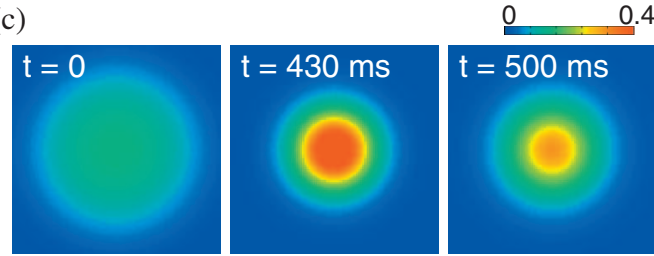

FIG. 1. (Color) (a) Time evolution of the normalized peak density $d_{1}+d_{2}$ and that of the fraction of atoms within $r=100 \mu \mathrm{m}$ for $a_{11}=0.75 \mathrm{~nm}, a_{22}=-1.5 \mathrm{~nm}, a_{12}=0$, and $\Omega=2 \pi \times 500 \mathrm{~Hz}$. The initial state is the ground state in the presence of a radial trapping potential with $\omega_{\perp}=2 \pi \times 25 \mathrm{~Hz}$, and the trapping potential is switched off at $t=0$. (b) Time scale of (a) is magnified. Smallamplitude oscillations in (b) are invisible in (a) because of the time resolution. (c) Snapshots of the normalized density profiles $\left(\left|\psi_{1}\right|^{2}\right.$ $\left.+\left|\psi_{2}\right|^{2}\right) \hbar /\left(m \omega_{\perp} N\right)$ at $t=0,430 \mathrm{~ms}$, and $500 \mathrm{~ms}$. The size of the images is $30 \mu \mathrm{m} \times 30 \mu \mathrm{m}$.

frequency for confinement in the $z$ direction is $\omega_{z}=2 \pi$ $\times 5 \mathrm{kHz}$. The ratio $\omega_{z} / \omega_{\perp}=200$ is sufficient for the $2 \mathrm{D}$ approximation to be valid. The number of atoms is $N=2500$, and the Rabi frequency is $\Omega=2 \pi \times 500 \mathrm{~Hz}$. The ratio $\omega_{z} / \Omega=10$ assures that no axial modes are excited by the Rabi oscillation. For this condition, the attractive interaction by $a_{22}$ dominates the kinetic pressure and the system collapses even in the absence of the trapping potential if the atomic state is fixed to the hyperfine state 2 .

Figure 1(a) shows the time evolution of normalized peak density $d_{1}+d_{2}$ and that of the fraction of atoms around the center, $N_{100 \mu \mathrm{m}}(t) / N$, where

$$
\begin{gathered}
d_{i}(t)=\frac{\hbar}{m \omega_{\perp} N}\left|\psi_{i}(r=0, t)\right|^{2} \quad(i=1,2), \\
N_{\rho}(t)=\sum_{i=1}^{2} \int_{0}^{\rho} 2 \pi r\left|\psi_{i}(r, t)\right|^{2} d r .
\end{gathered}
$$

We can clearly see that collapse is prevented and the BEC droplet is dynamically stabilized. The Rabi oscillation between the two hyperfine states induces oscillations of the total density at the same frequency, with the amplitude being 

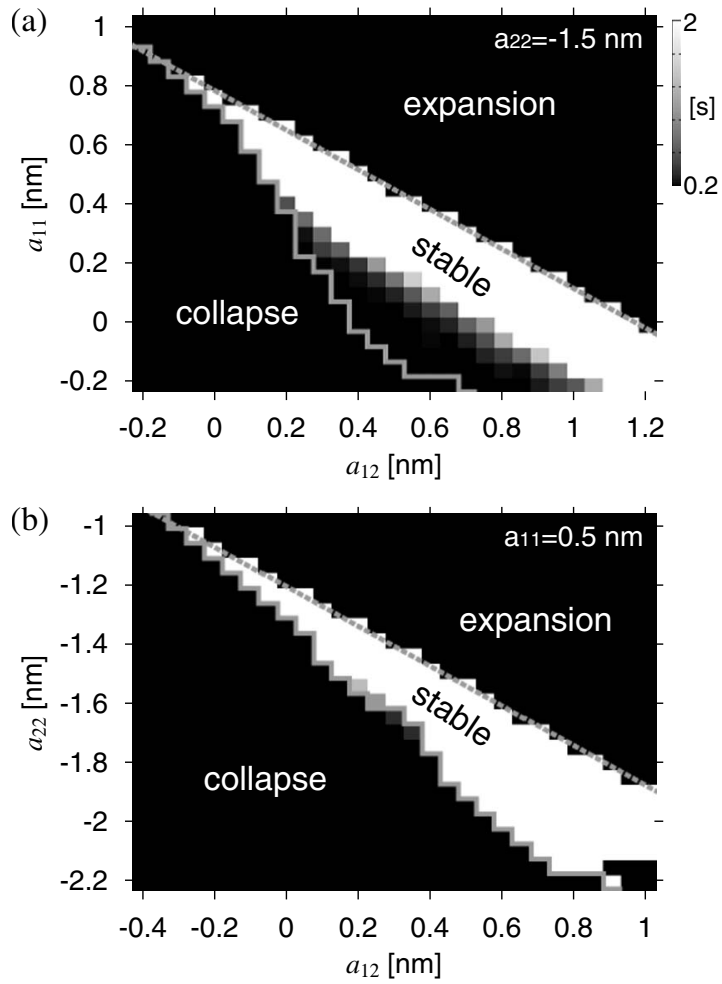

FIG. 2. Stability diagrams for a BEC droplet with respect to (a) $a_{12}$ and $a_{11}$ for $a_{22}=-1.5 \mathrm{~nm}$ and (b) $a_{12}$ and $a_{22}$ for $a_{11}=0.5 \mathrm{~nm}$. The gray scale shows the lifetime of the BEC droplet. The white region has a lifetime of at least $2 \mathrm{~s}$. The solid lines divide the regions of collapse and expansion. The dashed lines are given by Eq. (28)

larger for higher density [compare the left and right panels in Fig. 1(b)]. The long-period oscillation at the frequency of about $8 \mathrm{~Hz}$ persists for a long time [solid curve in Fig. 1(a)]. The initial decrease in the dashed curve in Fig. 1(a) shows that a small fraction of atoms are lost from the central region mainly during the initial formation of the droplet. These atoms have escaped from the droplet for lack of the radial trapping potential.

We next consider the stability of the BEC droplet for various values of scattering lengths. Figure 2(a) shows the stability diagram with respect to $a_{11}$ and $a_{12}$ for $a_{11}=-1.5 \mathrm{~nm}$ and Fig. 2(b) with respect to $a_{22}$ and $a_{12}$ for $a_{11}=0.5 \mathrm{~nm}$. In the white region, the lifetime of the BEC droplet is at least $2 \mathrm{~s}$. We find that a band of stable region lies between regions of collapse and expansion. These two types of instability are divided by the solid lines in Fig. 2. The stable region disappears for $a_{12} \lesssim-0.2 \mathrm{~nm}$. As we can see in Fig. 2(a), the value of $a_{11}$ in the stable region becomes negative as $a_{12}$ increases. However, this would not hinder an initial state of the condensate from being prepared. One can prepare the condensate in a positive $a_{11}$ and switch $a_{11}$ to an appropriate negative value at $t=0$.

The stability region is not sensitive to the Rabi frequency $\Omega$. To see this, we normalize the time, length, and wave function by $|\Omega|^{-1}$, $[\hbar /(m|\Omega|)]^{1 / 2}$, and $N[m|\Omega| / \hbar]^{1 / 2}$, respectively. GP equation (5a) then becomes

$$
\begin{aligned}
i \frac{\partial \psi_{1}}{\partial t}= & \left(-\frac{\nabla^{2}}{2}+\frac{\omega_{\perp}^{2}}{2|\Omega|^{2}} r^{2}\right) \psi_{1}+\psi_{2} \\
& +4 \pi N \sqrt{\frac{m \omega_{z}}{2 \pi \hbar}}\left(a_{11}\left|\psi_{1}\right|^{2} \psi_{1}+a_{12}\left|\psi_{2}\right|^{2} \psi_{1}\right)
\end{aligned}
$$

We note that $\Omega$ is included only in the term of the radial trapping potential used for preparing the initial state, and therefore the change in $\Omega$ only affects the initial wave function in the normalized form of GP equation (10). We performed numerical simulations also for $\Omega=2 \pi \times 1 \mathrm{kHz}$ and confirmed that the stability region is almost the same as in Fig. 2.

\section{ANALYTIC RESULTS}

In this section, we analytically study the stabilization of a BEC droplet shown in Sec. III.

We first transform wave functions $\psi_{1}$ and $\psi_{2}$ into

$$
\begin{gathered}
\Psi_{1}=\psi_{1} \cos |\Omega| t+i e^{i \phi} \psi_{2} \sin |\Omega| t, \\
\Psi_{2}=\psi_{2} \cos |\Omega| t+i e^{-i \phi} \psi_{1} \sin |\Omega| t,
\end{gathered}
$$

where $\Omega=e^{i \phi}|\Omega|$. Substituting Eq. (11) into GP equation (5), we find that the terms proportional to $|\Omega|$ vanish, and we obtain

$$
\begin{aligned}
i \hbar \frac{\partial \Psi_{1}}{\partial t}= & -\frac{\hbar^{2}}{2 m} \nabla^{2} \Psi_{1}+\frac{g_{11}+g_{22}}{8}\left(3\left|\Psi_{1}\right|^{2} \Psi_{1}+2\left|\Psi_{2}\right|^{2} \Psi_{1}\right. \\
& \left.-e^{2 i \phi} \Psi_{1}^{*} \Psi_{2}^{2}\right)+\frac{g_{12}}{4}\left(\left|\Psi_{1}\right|^{2} \Psi_{1}+2\left|\Psi_{2}\right|^{2} \Psi_{1}\right. \\
& \left.+e^{2 i \phi} \Psi_{1}^{*} \Psi_{2}^{2}\right)+\left(g_{11}-g_{22}\right)\left\{\frac{\cos 2|\Omega| t}{2}\left|\Psi_{1}\right|^{2} \Psi_{1}\right. \\
& \left.+\frac{i \sin 2|\Omega| t}{4}\left[e^{-i \phi} \Psi_{2}^{*} \Psi_{1}^{2}-e^{i \phi}\left(2\left|\Psi_{1}\right|^{2}+\left|\Psi_{2}\right|^{2}\right) \Psi_{2}\right]\right\} \\
& +\frac{g_{11}+g_{22}-2 g_{12}}{8}\left\{\operatorname { c o s } 4 | \Omega | t \left(\left|\Psi_{1}\right|^{2} \Psi_{1}-2\left|\Psi_{2}\right|^{2} \Psi_{1}\right.\right. \\
& \left.+e^{2 i \phi} \Psi_{1}^{*} \Psi_{2}^{2}\right)+i \sin 4|\Omega| t\left[e^{-i \phi} \Psi_{2}^{*} \Psi_{1}^{2}+e^{i \phi}\left(-2\left|\Psi_{1}\right|^{2}\right.\right. \\
& \left.\left.\left.+\left|\Psi_{2}\right|^{2}\right) \Psi_{2}\right]\right\},
\end{aligned}
$$

where we set $V_{1}=V_{2}=0$. The expression of $i \hbar \partial \Psi_{2} / \partial t$ is obtained by replacements $\Psi_{1} \leftrightarrow \Psi_{2}, g_{11} \leftrightarrow g_{22}$, and $e^{i \phi} \leftrightarrow e^{-i \phi}$ in Eq. (12).

The initial state of $\psi_{2}$ is assumed to be $\psi_{2}=0$ and then $\Psi_{2}=0$ at $t=0$. Setting $\Psi_{2}=0$ in the expression of $i \hbar \partial \Psi_{2} / \partial t$ gives

$$
\begin{aligned}
i \hbar \frac{\partial \Psi_{2}}{\partial t}= & \frac{i e^{-i \phi}}{8}\left[2\left(g_{11}-g_{22}\right) \sin 2|\Omega| t\right. \\
& \left.+\left(g_{11}+g_{22}-2 g_{12}\right) \sin 4|\Omega| t\right]\left|\Psi_{1}\right|^{2} \Psi_{1},
\end{aligned}
$$

which indicates that $\Psi_{2}$ is of the order of $1 /|\Omega|$. We therefore approximate that $\Psi_{2}$ is always zero. Equation (12) is then approximately reduced to the single-component GP equation 


$$
i \hbar \frac{\partial \Psi_{1}}{\partial t}=-\frac{\hbar^{2}}{2 m} \nabla^{2} \Psi_{1}+G(t)\left|\Psi_{1}\right|^{2} \Psi_{1}
$$

where

$$
G(t)=G_{0}+G_{1} \cos 2|\Omega| t+G_{2} \cos 4|\Omega| t,
$$

with

$$
\begin{gathered}
G_{0}=\frac{3\left(g_{11}+g_{22}\right)+2 g_{12}}{8}, \\
G_{1}=\frac{g_{11}-g_{22}}{2}, \\
G_{2}=\frac{g_{11}+g_{22}-2 g_{12}}{8} .
\end{gathered}
$$

To check if Eq. (14) captures the stabilization mechanism for a BEC droplet, we perform a variational analysis by using time-dependent Gaussian variational wave function [16]

$$
\Psi_{1}=\frac{\sqrt{N}}{\sqrt{\pi} R(t)} \exp \left[-\frac{r^{2}}{2 R^{2}(t)}+i \frac{m \dot{R}(t) r^{2}}{2 \hbar R(t)}\right],
$$

where $R(t)$ is the variational parameter that characterizes the size of the droplet and the second term in the exponent describes mass current which is required to satisfy the equation of continuity. The action that derives Eq. (14) has the form

$$
K=\int d \boldsymbol{r} d t\left[-i \hbar \Psi_{1}^{*} \frac{\partial}{\partial t} \Psi_{1}-\frac{\hbar^{2}}{2 m} \Psi_{1}^{*} \nabla^{2} \Psi_{1}+\frac{G(t)}{2}\left|\Psi_{1}\right|^{4}\right] .
$$

Substituting Eq. (19) into Eq. (20) and taking $\delta K / \delta R=0$, we obtain the equation of motion for $R(t)$,

$$
\ddot{R}(t)=\left[\frac{\hbar^{2}}{m^{2}}+\frac{N G(t)}{2 \pi m}\right] \frac{1}{R^{3}(t)} .
$$

Noting that the dynamics comprises rapid and slow oscillations as shown in Figs. 1(a) and 1(b), we separate $R(t)$ into a slowly varying part $R_{0}(t)$ and rapidly oscillating part $\varepsilon(t)$ as

$$
R(t)=R_{0}(t)+\varepsilon(t) .
$$

The rapidly oscillating part of Eq. (21) is approximated to be

$$
\ddot{\varepsilon}(t) \simeq \frac{N}{2 \pi m R_{0}^{3}(t)}\left(G_{1} \cos 2|\Omega| t+G_{2} \cos 4|\Omega| t\right),
$$

where we neglect the terms of order $|\Omega|^{-2}$. Since $R_{0}$ can be regarded as a constant in the fast time scale in Eq. (23), we have

$$
\varepsilon(t) \simeq-\frac{N}{32 \pi m R_{0}^{3}(t)|\Omega|^{2}}\left(4 G_{1} \cos 2|\Omega| t+G_{2} \cos 4|\Omega| t\right) .
$$

Substituting Eq. (24) into the slowly varying part of Eq. (21),

$$
\begin{aligned}
\ddot{R}_{0}(t)= & \left(\frac{\hbar^{2}}{m^{2}}+\frac{N G_{0}}{2 \pi m}\right) \frac{1}{R_{0}^{3}(t)}-\frac{3 N}{2 \pi m R_{0}^{4}(t)} \\
& \times \overline{\varepsilon(t)\left(G_{1} \cos 2|\Omega| t+G_{2} \cos 4|\Omega| t\right)}+O\left(\varepsilon^{2}\right),
\end{aligned}
$$

where the overbar indicates the time average for the rapid oscillation, we finally obtain an effective equation of motion for slowly varying parameter $R_{0}(t)$ as

$$
\ddot{R}_{0}(t) \simeq\left(\frac{\hbar^{2}}{m^{2}}+\frac{N G_{0}}{2 \pi m}\right) \frac{1}{R_{0}^{3}(t)}+\frac{3 N^{2}\left(4 G_{1}^{2}+G_{2}^{2}\right)}{128 \pi m^{2}|\Omega|^{2} R_{0}^{7}(t)} .
$$

The first term on the right-hand side of Eq. (26) originates from the kinetic energy and the constant part of the interaction energy. For stationary $R_{0}$ to exist, the coefficient of the first term must be negative, i.e.,

$$
\frac{m N G_{0}}{2 \pi \hbar^{2}}<-1
$$

which gives the condition for stability against expansion. Using Eqs. (16) and (27), the boundary between the stable and unstable regions is given by

$$
3\left(a_{11}+a_{22}\right)+2 a_{12}=-\frac{4}{N} \sqrt{\frac{2 \pi \hbar}{m \omega_{z}}} .
$$

This boundary is shown in Fig. 2 by the dashed lines, which show close agreement with the numerically obtained boundary.

We note that the second term on the right-hand side of Eq. (26), which originates from the oscillating part of the interaction in Eq. (14), prevents the system from collapsing. Thus, the effective oscillation of the interaction by the Rabi oscillation dynamically stabilizes the BEC droplet. The system is stationary $\left(\ddot{R}_{0}=0\right)$ with the size of the droplet,

$$
R_{0}=\left[\frac{3 N^{2}\left(4 G_{1}^{2}+G_{2}^{2}\right)}{64 \pi|\Omega|^{2}\left|2 \pi \hbar^{2}+m N G_{0}\right|}\right]^{1 / 4},
$$

which is $\simeq 6.4 \mu \mathrm{m}$ for the parameters in Fig. 1. Comparing the density profile at $t=0\left[R=\left(\hbar / m \omega_{\perp}\right)^{1 / 2} \simeq 7.6 \mu \mathrm{m}\right]$ with those at $t=430$ and $500 \mathrm{~ms}$ in Fig. 1(c), we can see that this value of $R_{0}$ agrees well with the numerical one. The frequency of the small oscillation around Eq. (29) is obtained as

$$
\omega=\frac{8 \sqrt{2}|\Omega|\left|2 \pi \hbar^{2}+m N G_{0}\right|}{\sqrt{3} m N \sqrt{4 G_{1}^{2}+G_{2}^{2}}},
$$

which is $\sim 2 \pi \times 12 \mathrm{~Hz}$ for the parameters in Fig. 1, in qualitative agreement with the numerical result of about $2 \pi$ $\times 8 \mathrm{~Hz}$. The difference may be due to the large amplitude of the oscillation as shown in Fig. 1(a).

\section{CONCLUSIONS AND DISCUSSION}

We have studied a 2D BEC of atoms in two hyperfine states which undergo Rabi oscillation by an external field. When scattering lengths $a_{11}, a_{22}$, and $a_{12}$ are different, the Rabi oscillation leads to effective oscillation of the interac- 
tion. If the scattering lengths fall in the stable regions such as those in Fig. 2, the self-trapped BEC in free space-a BEC droplet-can be stabilized by the mechanism discussed in Refs. [4,5].

Solving the two-component GP equation (5) numerically, we have shown that the stabilization mechanism works and the BEC droplet is stabilized (Fig. 1). The stabilization is shown to be possible even if the system starts from the ground state in a radial trapping potential, which is switched off at $t=0$. We have obtained the stability diagram with respect to scattering lengths (Fig. 2), in which there exists the stability region between the regions of collapse and expansion.

To elucidate the mechanism for the dynamic stabilization of a BEC droplet, we approximately reduced the twocomponent GP equation to a single component equation (14) and studied it by using the Gaussian variational method. We found that the Rabi oscillation generates an effective potential that counteracts the attractive interaction, thereby creating a stable droplet state. The size of the droplet and its breathing-mode oscillation frequency are obtained by the variational analysis, which produces results in qualitative agreement with the numerical ones.

Our scheme applies to any system where atoms in the two relevant hyperfine states are not transferred to other states by inelastic collisions and the coupling field is available that makes Rabi oscillations between the two states. For example, the hyperfine states $\left|F, m_{F}\right\rangle=|1,1\rangle$ and $|2,2\rangle$ of ${ }^{87} \mathrm{Rb}$ and ${ }^{23} \mathrm{Na}$ and $|2,2\rangle$ and $|3,3\rangle$ of ${ }^{85} \mathrm{Rb}$ are possible candidates if their scattering lengths can be tuned to fall in the stable region. Unfortunately, for $|1,1\rangle$ and $|2,2\rangle$ hyperfine states of

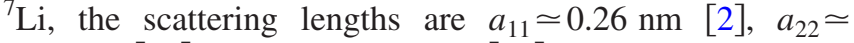
$-1.4 \mathrm{~nm}$ [17], and $a_{12} \simeq-1.4 \mathrm{~nm}$ [18], and this set of scattering lengths is located out of the stability region. By systematic calculations as in Fig. 2, we found that there is no stable set of $a_{11}$ and $a_{22}$ for this value of $a_{12}$.

Using the above pairs of hyperfine states, inelastic decay of the upper hyperfine state occurs only through dipoledipole interaction. The dipolar decay rate $K$ is typically less than $10^{-14} \mathrm{~cm}^{3} / \mathrm{s}$ [19], and the peak density in Fig. 1(a) is $n \simeq 0.4 N m \omega_{\perp} / \hbar\left[m \omega_{z} /(\pi \hbar)\right]^{1 / 2}$, which gives $n K \lesssim 0.2 \mathrm{~s}^{-1}$. Therefore, the lifetime of the droplet in the presence of the dipolar loss is estimated to be more than $5 \mathrm{~s}$.

Another possibility is the use of two states in the same hyperfine manifold, e.g., $|1,1\rangle$ and $|1,0\rangle$, in which modest magnetic fields $(\sim 1-10 \mathrm{G})$ should be applied to suppress the spin-exchange collisions $(|1,0\rangle+|1,0\rangle \rightarrow|1,1\rangle+|1,-1\rangle)$. For example, for these states of ${ }^{7} \mathrm{Li}$, there are regions in which Eq. (28) becomes small and negative as a function of magnetic field, suggesting that stabilization is possible.

The 2D potential may not be perfectly flat in experiments, and a residual weak potential in $2 \mathrm{D}$ - e.g., a weak harmonic potential-may exist. Even in this case, we can distinguish self-trapping from trapping by a residual potential if the size of a droplet is made much smaller than the scale of inhomogeneity in the 2D potential.

As in the case of oscillating the scattering length [4,5], we have not been able to create a stable BEC droplet in $3 \mathrm{D}$ in the absence of dissipation. One reason for this difficulty is that collapse is stronger for higher dimensions [3], and therefore the amplitude of the rapid oscillation should be larger to suppress the collapse. However, this breaks the smallamplitude and Gaussian approximations, and unstable modes are dynamically induced.

The present method is different from the one in Refs. $[4,5]$, in that explicit oscillation of the interaction using the Feshbach resonance is not needed. The rapid modulation of the applied magnetic field for the Feshbach resonance may cause some experimental difficulties-e.g., an induced current in the apparatus and resultant heating - and therefore the present method is expected to facilitate the experimental realization of a BEC droplet.

Note added in proof. A recent paper by $\mathrm{H}$. Susanto et al. [20] discusses a model similar to ours but in the regime of relatively small $\Omega$. In that case, they find that constant $\Omega$ cannot stabilize a BEC droplet in 2D.

\section{ACKNOWLEDGMENTS}

This work was supported by Grants-in-Aid for Scientific Research (Grant Nos. 17740263 and 17071005) and by the 21st Century COE programs on "Coherent Optical Science" and "Nanometer-Scale Quantum Physics" from the Ministry of Education, Culture, Sports, Science and Technology of Japan. The work at Rice was supported by the NSF, ONR, and the Welch Foundation (Grant No. C-1133). H.S. acknowledges support by the Matsuo Foundation.
[1] L. Khaykovich, F. Schreck, G. Ferrari, T. Bourdel, J. Cubizolles, L. D. Carr, Y. Castin, and C. Salomon, Science 296, 1290 (2002).

[2] K. E. Strecker, G. B. Partridge, A. G. Truscott, and R. G. Hulet, Nature (London) 417, 150 (2002).

[3] C. Sulem and P. L. Sulem, The Nonlinear Schrödinger Equation (Springer, New York, 1999).

[4] H. Saito and M. Ueda, Phys. Rev. Lett. 90, 040403 (2003).

[5] F. Kh. Abdullaev, J. G. Caputo, R. A. Kraenkel, and B. A. Malomed, Phys. Rev. A 67, 013605 (2003).

[6] See, for example, R. P. Feynman, R. B. Leighton, and M. L.
Sands, The Feynman Lectures on Physics (Addison-Wesley, Reading, 1964), Vol. II, Chap. 29.

[7] P. G. Kevrekidis, G. Theocharis, D. J. Frantzeskakis, and B. A. Malomed, Phys. Rev. Lett. 90, 230401 (2003).

[8] G. D. Montesinos, V. M. Pérez-García, and P. J. Torres, Physica D 191, 193 (2004).

[9] G. D. Montesinos, V. M. Pérez-García, and H. Michinel, Phys. Rev. Lett. 92, 133901 (2004).

[10] M. Matuszewski, E. Infeld, B. A. Malomed, and M. Trippenbach, Phys. Rev. Lett. 95, 050403 (2005); M. Trippenbach, M. Matuszewski, and B. A. Malomed, Europhys. Lett. 70, 8 
(2005)

[11] A. Itin, T. Morishita, and S. Watanabe, Phys. Rev. A 74, 033613 (2006).

[12] B. A. Malomed, Soliton Management in Periodic Systems (Springer, New York, 2006).

[13] H. Saito and M. Ueda, Phys. Rev. A 70, 053610 (2004).

[14] H. Saito and M. Ueda, Phys. Rev. A 74, 023602 (2006).

[15] S. Inouye, M. R. Andrews, J. Stenger, H.-J. Miesner, D. M. Stamper-Kurn, and W. Ketterle, Nature (London) 392, 151 (1998).

[16] V. M. Pérez-García, H. Michinel, J. I. Cirac, M. Lewenstein, and P. Zoller, Phys. Rev. Lett. 77, 5320 (1996); Phys. Rev. A 56, 1424 (1997).

[17] E. R. I. Abraham, W. I. McAlexander, C. A. Sackett, and R. G. Hulet, Phys. Rev. Lett. 74, 1315 (1995).

[18] W. I. McAlexander, Ph.D. thesis, Rice University (2000).

[19] A. J. Moerdijk and B. J. Verhaar, Phys. Rev. A 53, R19 (1996); H. M. J. M. Boesten, A. J. Moerdijk, and B. J. Verhaar, ibid. 54, R29 (1996).

[20] H. Susanto, P. G. Kevrekidis, B. A. Malomed, and F. Kh. Abdullaev, Phys. Lett. A (to be published). 